\title{
Aberrant cerebral blood flow in tinnitus patients with migraine: a perfusion functional MRI study
}

\author{
Zhen-Gui $\mathrm{Xu}^{1+}$, Jin-Jing $\mathrm{Xu}^{2+}, \mathrm{Yu}$-Chen $\mathrm{Chen}{ }^{3}$, Jinghua $\mathrm{Hu}^{2}$, Yuanqing $\mathrm{Wu}^{2^{*}}$ and Yuan Xue ${ }^{1^{*}}$
}

\begin{abstract}
Purpose: Migraine is often accompanied with chronic tinnitus that will affect the cerebral blood flow (CBF) and exacerbate the tinnitus distress. However, the potential relationship between migraine and tinnitus remains unclear. This study will investigate whether aberrant CBF patterns exist in migraine patients with tinnitus and examine the influence of migraine on CBF alterations in chronic tinnitus.

Materials and methods: Participants included chronic tinnitus patients $(n=45)$ and non-tinnitus controls $(n=50)$, matched for age, sex, education, and hearing thresholds. CBF images were collected and analyzed using arterial spin labeling (ASL) perfusion functional magnetic resonance imaging (fMRI). Regions with major CBF differences between tinnitus patients and non-tinnitus controls were first detected. The effects of migraine on tinnitus for CBF alterations were further examined. Correlation analyses illustrated the association between CBF values and tinnitus severity as well as between CBF and severity of migraine.
\end{abstract}

Results: Compared with non-tinnitus controls, chronic tinnitus patients without migraine exhibited decreased CBF, primarily in right superior temporal gyrus (STG), bilateral middle frontal gyrus (MFG), and left superior frontal gyrus (SFG); decreased CBF in these regions was correlated with tinnitus distress. There was a significant effect of migraine on tinnitus for CBF in right STG and MFG. Moreover, the severity of migraine correlated negatively with CBF in tinnitus patients.

Conclusions: Chronic tinnitus patients exhibited reduced CBF in the auditory and prefrontal cortex. Migraine may facilitate a CBF decrease in the setting of tinnitus, which may underlie the neuropathological mechanisms of chronic tinnitus comorbid with migraine.

Keywords: migraine, chronic tinnitus, cerebral blood flow, functional MRI

\footnotetext{
*Correspondence: 15366110097@163.com; tz_dsh@163.com

${ }^{2}$ Department of Otolaryngology, Nanjing First Hospital, Nanjing Medical

University, No.68, Changle Road, 210006 Nanjing, China

'Department of Otolaryngology, Nanjing Pukou Central Hospital, Pukou

Branch Hospital of Jiangsu Province Hospital, No.166, Shanghe Road, 211899

Nanjing, China

Full list of author information is available at the end of the article
}

(C) The Author(s). 2021 Open Access This article is licensed under a Creative Commons Attribution 4.0 International License, which permits use, sharing, adaptation, distribution and reproduction in any medium or format, as long as you give appropriate credit to the original author(s) and the source, provide a link to the Creative Commons licence, and indicate if changes were made. The images or other third party material in this article are included in the article's Creative Commons licence, unless indicated otherwise in a credit line to the material. If material is not included in the article's Creative Commons licence and your intended use is not permitted by statutory regulation or exceeds the permitted use, you will need to obtain permission directly from the copyright holder. To view a copy of this licence, visit http://creativecommons.org/licenses/by/4.0/ The Creative Commons Public Domain Dedication waiver (http://creativecommons.org/publicdomain/zero/1.0/) applies to the data made available in this article, unless otherwise stated in a credit line to the data. 


\section{Introduction}

Tinnitus is a common auditory disorder that affects approximately $10-15 \%$ of adult populations, which severely impairs life quality of about $1-2 \%$ of the general population [1, 2]. Chronic tinnitus patients suffer from secondary tinnitus symptoms or comorbidities, such as depression, anxiety, insomnia, and chronic pain [3, 4]. Furthermore, nearly $26-47 \%$ of patients with tinnitus also suffer from migraine or headache[5]. The association between tinnitus and migraine has been described in prior studies. Therefore, migraine is a risk factor that may play an important role for tinnitus-related impairment in quality of life. It has been proposed that tinnitus and migraine overlap in their pathophysiological mechanism by sharing specific alterations in thalamocortical activity[6, 7]. However, the potential relationship between migraine and tinnitus still remains unclear.

Previous researches using functional magnetic resonance imaging (fMRI) have suggested that tinnitus is associated with aberrant brain functional changes in temporal cortex and non-auditory brain areas, including the prefrontal cortex, parahippocampus, insula and cerebellum[8]. Moreover, researches using cerebral perfusion, investigated via single-photon emission computed tomography (SPECT) and positron emission tomography (PET), showed that tinnitus patients exhibit decreased or increased cerebral blood flow (CBF) in widespread brain regions, such as temporal cortex, prefrontal cortex, and parahippocampal gyrus [9-11]. Arterial spin labeling (ASL) is used to evaluate CBF at resting state and could serve as a marker of functional activation albeit, which achieves a direct measure of regional CBF and independent of complicated calculations [12]. ASL perfusion fMRI, with higher resolution and accurate localization, has been applied to detect the CBF in various neurological or psychiatry disorders [13]. However, Emmert et al. did not observe any significant $\mathrm{CBF}$ changes between tinnitus patients and healthy controls using ASL [14], probably due to the limited sample size. Regarding migraine, previous studies have found that migraine patients exhibited hypoperfusion, hyperperfusion or no changes in whole-brain CBF [15-17]. Therefore, further research is required to investigate what regions reveal altered CBF in chronic tinnitus and to determine whether migraine is involved in the fluctuation of $\mathrm{CBF}$ values in these regions.

To address this issue, we raise the hypothesis that chronic tinnitus would exhibit aberrant CBF compared with non-tinnitus controls and that the migraine would have potential effect on the CBF in tinnitus patients. This study aimed to assess CBF differences between chronic tinnitus patients and matched controls using ASL perfusion $\mathrm{AMRI}$ and observed the effect of migraine on CBF changes in chronic tinnitus patients. We hypothesized that tinnitus patients would exhibit abnormal CBF in specific brain regions and migraine would exacerbate $\mathrm{CBF}$ abnormalities on tinnitus patients.

\section{Materials and methods \\ Subjects and clinical data}

Study protocol was approved by the Research Ethics Committee of the Nanjing Medical University prior to study initiation. All the subjects provided written informed consent before any study procedures. Ninety-five subjects, including 45 patients with chronic bilateral tinnitus and 50 non-tinnitus controls (all right-handed and completed at least 8 years of education), were recruited through community health screening and newspaper advertisements and matched for sex, age, and education. According to the International Classification of Headache Disorders, Third Edition (beta version) (ICHD - 3 beta)[18], migraineurs without aura were diagnosed by a neurologist, then tinnitus patients were divided into two groups [ 20 with migraine (5 with chronic migraine and 15 with episodic migraine), and 25 without migraine]. Of the 20 patients with migraine headaches, 8 had headaches preferentially located at the right side of the head, 7 at the left side and 5 had bilateral headaches, or no side preference. The disease duration and attack frequency of migraine were also recorded. For the controls, they should have no personal or family history of migraine or other headaches. The Iowa version of the Tinnitus Handicap Questionnaires (THQ) [19] as well as a pure tone audiometry (PTA) examination was used to assess the tinnitus severity, tinnitus distress, and the hearing threshold. Any participants who had hearing loss (defined as thresholds $\geq 25 \mathrm{~dB} \mathrm{HL}$ ) at the frequencies of $0.25 \mathrm{kHz}, 0.5 \mathrm{kHz}, 1 \mathrm{kHz}, 2 \mathrm{kHz}, 4 \mathrm{kHz}$, and $8 \mathrm{kHz}$ were excluded from the current study. Participants were excluded if they suffered from pulsatile tinnitus, hyperacusis or Meniere's diseases, or if they had a past history of alcoholism, stroke, migraine, brain injury, anemia, Alzheimer's disease, Parkinson's disease, epilepsy, major depression or other neurological or psychiatric illness, MRI contraindications or severe visual loss, thyroid dysfunction, cancer, severe heart diseases and damaged liver/ kidney function.

According to the Self-Rating Depression Scale (SDS) and the Self-Rating Anxiety Scale (SAS) (overall scores < 50 , respectively), none of the participants had depression or anxiety. The pain intensity of migraine was measured by the visual analogue scale (VAS) and severity of migraine was measured by the Headache Impact Test- 6 (HIT-6).

\section{MRI data acquisition}

All participants were scanned using a 3.0 T MRI scanner (Ingenia, Philips Medical Systems, Netherlands). Foam 
Table 1 Demographic and clinical characteristics of tinnitus patients and non-tinnitus controls

\begin{tabular}{llll}
\hline & $\begin{array}{l}\text { Tinnitus patients } \\
(\boldsymbol{n}=\mathbf{4 5})\end{array}$ & $\begin{array}{l}\text { Non-tinnitus controls } \\
(\boldsymbol{n}=\mathbf{5 0 )}\end{array}$ & $\boldsymbol{p}$ value \\
\hline Age (years) & $49.82 \pm 11.51$ & $47.36 \pm 12.39$ & 0.320 \\
Gender (male: female) & 15.30 & $20: 30$ & 0.501 \\
Education levels (years) & $12.56 \pm 2.95$ & $12.84 \pm 3.13$ & 0.651 \\
Tinnitus duration (months) & $39.20 \pm 37.94$ & - & - \\
THQ score & $52.01 \pm 13.77$ & -- & - \\
HIT-6 score & $62.00 \pm 2.36$ & -- & - \\
VAS score & $5.50 \pm 1.43$ & $16.95 \pm 2.49$ & - \\
Hearing thresholds (left) & $16.06 \pm 2.65$ & $17.00 \pm 2.34$ & 0.094 \\
Hearing thresholds (right) & $16.52 \pm 3.03$ & $16.92 \pm 1.62$ & 0.385 \\
Hearing thresholds (average) & $16.24 \pm 2.52$ & 0.126 \\
\hline Da a
\end{tabular}

Data are represented as Mean \pm SD. The PTA from both ears was averaged. PTA, puretone audiometry; THQ, Tinnitus Handicap Questionnaires; HIT-6, migraine impact test-6; VAS, visual analogue scale

padding and earplugs were used to reduce the head motion and scanner noise. The participants were instructed to rest quietly with their eyes closed and avoiding either falling asleep or making sudden head motions, and to not think of anything in particular during MRI scan. The subjects with migraine were not having pain during MRI scan. High resolution T1-weighted images were acquired using three-dimensional turbo fast echo (3DTFE) sequence as follows: repetition time $(\mathrm{TR})=8.1 \mathrm{~ms}$, echo time $(\mathrm{TE})=3.7 \mathrm{~ms}$, thickness $=1 \mathrm{~mm}$, slices $=170$, gap $=0 \mathrm{~mm}$, flip angle $(\mathrm{FA})=8^{\circ}$, field of view $(\mathrm{FOV})=$ $256 \times 256 \mathrm{~mm}^{2}$, and acquisition matrix $=256 \times 256$. The structural sequence took $5 \mathrm{~min}$ and $29 \mathrm{~s}$. ASL images were obtained with a pseudo-continuous ASL (pcASL) sequence with a 2D fast spin-echo acquisition and background suppression using the parameters as follows: $\mathrm{TR}=4000 \mathrm{~ms}, \mathrm{TE}=11 \mathrm{~ms}$, slice thickness $=4 \mathrm{~mm}$, label duration $=1650 \mathrm{~ms}$; post-label delay $=2000 \mathrm{~ms}$, $\mathrm{FA}=90^{\circ}, \quad \mathrm{FOV}=220 \times 220 \mathrm{~mm}^{2}$, slices thickness $=4$ $\mathrm{mm}$, gap $=0.4 \mathrm{~mm}$, matrix $=64 \times 64$. The ASL sequence took $4 \mathrm{~min}$ and $18 \mathrm{~s}$.

\section{Imaging data processing}

A voxel-based morphometry (VBM) approach was performed to estimate whole brain volumes using the VBM8 toolbox (http://dbm.neuro.uni-jena.de/vbm). DARTEL was used to improve inter-subject registration of the structural images. Briefly, cerebral tissues were segmented into gray matter (GM), white matter (WM), and cerebrospinal fluid by a unified segmentation algorithm [20]. Then, resulting GM and WM images were normalized to the MNI template, followed by smoothing using an 8-mm full width at half maximum (FWHM) Gaussian kernel. Finally, the resulting voxel-wise GM volume maps were entered as covariates in the ASL data analysis.

The ASL data were preprocessed to generate CBF maps using the ASL Perfusion MRI Signal Processing Toolbox (ASLtbx), which is based on SPM12 (http://www.fil.ion. ucl.ac.uk/spm/) [21]. All images were first rearranged and adjusted to correct head movement. Next, a nonlinear transformation was performed on the CBF images of healthy controls, which were co-registered with the PETperfusion template in Montreal Neurological Institute (MNI) space. The MNI-standard CBF template was defined as the average co-registered CBF images of healthy controls. The CBF images of all participants were then coregistered to the MNI-standard CBF template. Every coregistered $\mathrm{CBF}$ was removed from the non-brain tissue. Then a spatial smoothing with an isotropic Gaussian at FWHM of $6 \mathrm{~mm}^{3}$ was followed. Finally, normalization was performed by dividing the CBF per voxel by the average $\mathrm{CBF}$ across the entire brain [22]. The relative $\mathrm{CBF}$ $(\mathrm{rCBF})$ was used in the whole analysis. None of the participants was excluded from the study due to head movement exceeding $2.0 \mathrm{~mm}$ of maximum translation in any of the $\mathrm{x}, \mathrm{y}$, and $\mathrm{z}$ directions or $2.0^{\circ}$ of the maximum rotation around the three axes.

\section{Statistical analyses}

Clinical measures were analyzed using Statistical Package for Social Sciences (SPSS) statistics software package version 20.0 (IBM Corp., Armonk, NY, USA). The

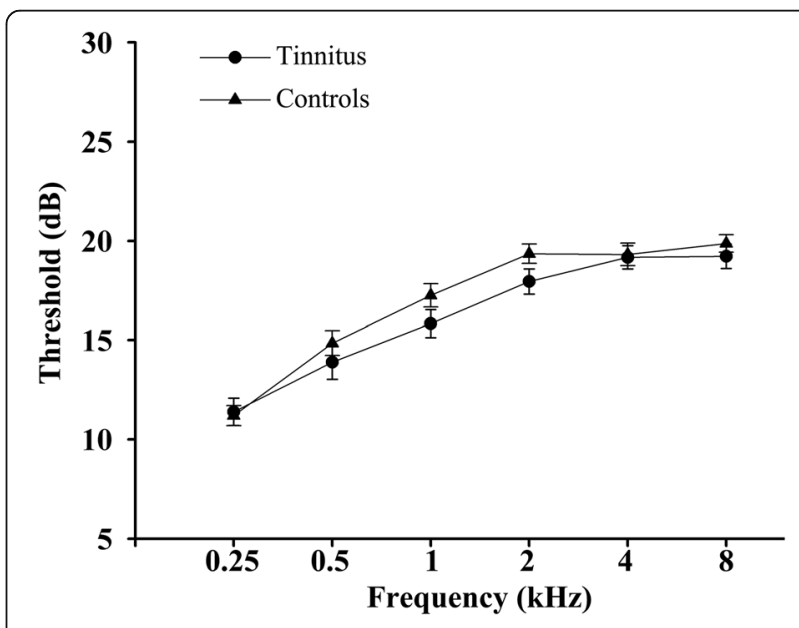

Fig. 1 Mean hearing thresholds of the chronic tinnitus patients and non-tinnitus controls. Data are presented as Mean \pm SEM 
Table 2 Demographic and clinical characteristics of tinnitus patients with and without migraine

\begin{tabular}{llll}
\hline & $\begin{array}{l}\text { Tinnitus with migraine } \\
(\boldsymbol{n}=\mathbf{2 0})\end{array}$ & $\begin{array}{l}\text { Tinnitus without migraine } \\
(\boldsymbol{n}=\mathbf{2 5})\end{array}$ & $\boldsymbol{p}$ value \\
\hline Age (years) & $49.00 \pm 12.26$ & $50.48 \pm 11.08$ & 0.673 \\
Gender (male: female) & $5: 15$ & $10: 15$ & 0.289 \\
Education levels (years) & $12.60 \pm 3.10$ & $12.52 \pm 2.89$ & 0.929 \\
Tinnitus duration (months) & $49.20 \pm 41.14$ & $31.20 \pm 33.90$ & 0.115 \\
THQ score & $56.78 \pm 12.98$ & $48.20 \pm 13.42$ & $0.036^{*}$ \\
HIT-6 score & $62.00 \pm 2.36$ & - & - \\
VAS score & $5.50 \pm 1.43$ & - & - \\
Migraine duration (months) & $42.40 \pm 35.92$ & - & - \\
Frequency (d/m) & $8.75 \pm 2.92$ & - & - \\
Hearing thresholds (left) & $16.59 \pm 2.80$ & $15.63 \pm 2.51$ & 0.230 \\
Hearing thresholds (right) & $16.54 \pm 2.71$ & $16.50 \pm 3.32$ & 0.964 \\
Hearing thresholds (average) & $16.17 \pm 2.04$ & $16.29 \pm 2.88$ & 0.869 \\
\hline
\end{tabular}

Data are represented as Mean \pm SD. The PTA from both ears was averaged. PTA, puretone audiometry; THQ, Tinnitus Handicap Questionnaires; HIT-6, migraine impact test-6; VAS, visual analogue scale. ${ }^{*} p<0.05$

statistical significance level was set at $p<0.05$, twotailed. One-way analysis of variance (ANOVA) was used to calculate the difference among the three groups followed by a post hoc test (t-test for means and $x^{2}$-test for proportions) between tinnitus patients with migraine and without migraine. For some non-normal distributed parameters, such as PTA, we used Mann-Whitney U test for between-group analysis.

A one-way analysis of variance (ANOVA) was then performed to determine between-group differences in brain volumes, with age, gender, and education as the nuisance covariates. Between-group rCBF differences were also calculated via one-way ANOVA in SPM12 with age, gender, education level and GM volume as the nuisance covariates. Significant thresholds were corrected using false discovery rate (FDR) criterion and set at $p<0.01$. A full-factorial model was utilized to detect potential interaction effects of tinnitus and migraine on rCBF differences. Full factorial analysis was conducted to analyse the main effects and interaction effect of tinnitus and migraine using SPM12 software. Specifically, the between-subject factors included tinnitus group and migraine group. Significant thresholds were corrected using cluster-level family-wise error (FWE), and the threshold was set at $p<0.01$.

The relationships between aberrant $\mathrm{rCBF}$ and each clinical characteristic were further investigated. Firstly, regions showing significant differences between groups were extracted. Then the mean $\mathrm{z}$-values of aberrant rCBF region mask were calculated within every subject. Pearson correlation analysis between the mean z-values and each clinical characteristic were performed using SPSS software. Partial correlations were calculated with age, sex, education, GM volume, and average hearing thresholds as the nuisance covariates. $P<0.05$ was considered statistically significant.

\section{Results}

\section{Demographics and clinical data}

Demographics and clinical characteristic data of all the chronic tinnitus patients and non-tinnitus controls were summarized in Table 1. No significant differences were found in terms of age, gender, education level, and hearing thresholds. Moreover, there were no significant differences in each frequency of auditory thresholds between two groups (Fig. 1). Furthermore, no significant differences were observed in terms of age, gender, education level, and hearing thresholds between tinnitus patients with and without migraine (Table 2). However, tinnitus patients with migraine had worse THQ scores than patients without migraine $(p<0.05)$.

\section{Structural data}

There were no significant differences in the comparisons of the whole-brain volumes (GM volume, WM volume and brain parenchyma volume) between chronic tinnitus

Table 3 Comparisons of volumes between tinnitus patients and non-tinnitus controls

\begin{tabular}{llll}
\hline Brain volume & Tinnitus patients & Non-tinnitus controls & $p$ value \\
\hline Gray matter volume (\% of TIV) & $31.78 \pm 2.01$ & $32.39 \pm 2.01$ & 0.143 \\
White matter volume (\% of TIV) & $29.59 \pm 1.54$ & $29.53 \pm 1.62$ & 0.861 \\
Brain parenchyma volume (\% of TIV) & $61.37 \pm 2.95$ & $61.92 \pm 3.16$ & 0.382 \\
\hline
\end{tabular}

Data are presented as mean \pm SD. TIV, total intracranial volume. 


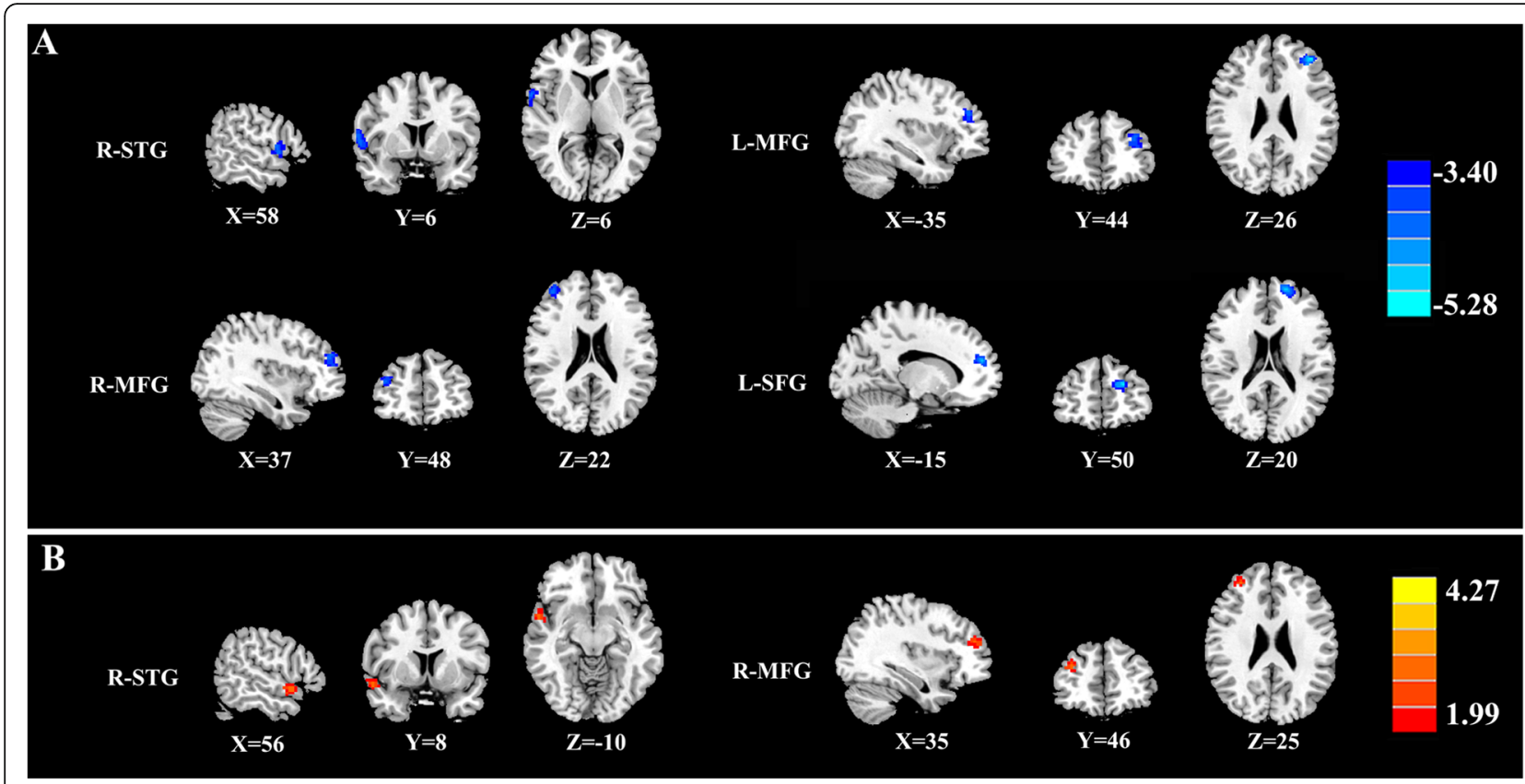

Fig. 2 The rCBF differences between the chronic tinnitus patients and non-tinnitus controls. (A) The tinnitus patients exhibited decreased rCBF in the right superior temporal gyrus (STG), left and right middle frontal gyrus (MFG), and left superior frontal gyrus (SFG) $(P<0.01$, FWE corrected); (B) The interaction effect of migraine and tinnitus in the right STG and right MFG $(P<0.01$, FWE corrected)

patients and controls (Table 3). Moreover, we also observed no significant differences of whole-brain volumes between tinnitus patients with migraine and without migraine. After Monte Carlo simulation correction, no suprathreshold voxel-wise difference in the GM and WM volumes between chronic tinnitus patients and controls was observed.

\section{Group rCBF differences}

The rCBF differences between the chronic tinnitus patients without migraine and non-tinnitus controls were shown in Fig. $2 \mathrm{~A}$ and Table 4. The tinnitus patients exhibited decreased $\mathrm{rCBF}$, primarily in the right superior temporal gyrus (STG), bilateral middle frontal gyrus (MFG), and left superior frontal gyrus (SFG) $(P<0.01$, FWE corrected). These reductions are due solely to the presence of tinnitus and not to the presence of subjects with migraine and not in the controls. The interaction effect of migraine on tinnitus was significant in the right STG and right MFG (Fig. 2B; Table 5) $(P<0.01$, FWE corrected). When the tinnitus patients had migraine, the rCBF would be decreased, especially in the right STG and right MFG. The rCBF values for different groups were shown in Fig. 3.

\section{Correlation analysis}

The significant correlations between the rCBF changes and the clinical data were depicted in Fig. 4. Regarding the tinnitus characteristics, the rCBF in the right STG and the right MFG was negatively associated with THQ scores, respectively $(\mathrm{r}=-0.333, p=0.036 ; \mathrm{r}=-0.486, p=0.001)$. Regarding the correlations between migraine and $\mathrm{rCBF}$ values, we observed that the HIT-6 scores were inversely correlated with the $\mathrm{rCBF}$ in the right STG $(\mathrm{r}=-0.597, p=0.019)$. Moreover, the VAS scores were inversely correlated with the $\mathrm{rCBF}$ in the right MFG $(\mathrm{r}=-0.652, p=0.008)$.

Table 4 Brain regions with significant differences in CBF between tinnitus without migraine and non-tinnitus controls

\begin{tabular}{llll}
\hline Brain regions & BA & Peak MNI coordinates $\mathbf{x}, \mathbf{y , ~} \mathbf{z}(\mathbf{m m})$ & Peak T value \\
\hline R superior temporal gyrus & 22 & $58,6,6$ & -4.3271 \\
$\mathrm{~L}$ middle frontal gyrus & 10 & $-35,44,26$ & -4.8734 \\
R middle frontal gyrus & 10 & $37,48,22$ & 159 \\
$\mathrm{~L}$ superior frontal gyrus & 10 & $-15,50,20$ & -4.2989 \\
\hline
\end{tabular}

Thresholds were set at a corrected $p<0.01$ corrected by FWE criterion 
Table 5 Regions showing interaction effects of tinnitus and migraine on CBF

\begin{tabular}{lllll}
\hline Brain regions & BA & Peak $\mathbf{M N I}$ coordinates $\mathbf{x}, \mathbf{y}, \mathbf{z}(\mathbf{m m})$ & Peak T value & Cluster size (Voxels) \\
\hline R superior temporal gyrus & 22 & $56,8,-10$ & 3.8773 & 109 \\
R middle frontal gyrus & 10 & $35,46,25$ & 3.9324 & 95 \\
\hline
\end{tabular}

Thresholds were set at a corrected $p<0.01$ corrected by FWE criterion

\section{Discussion}

The current study explored for the first time the associations between migraine and tinnitus using ASL perfusion fMRI. Compared to non-tinnitus controls, tinnitus patients exhibited reduced CBF in temporal and prefrontal cortex, which was associated with tinnitus distress. Interestingly, we also observed that migraine exacerbated $\mathrm{rCBF}$ reduction in tinnitus patients, and the severity of migraine was linked with decreased $\mathrm{rCBF}$.

Chronic tinnitus has been associated with GM changes in widespread brain regions [23]. We expected to observe brain atrophy in these patients, however, neither regional nor whole-brain atrophy was observed in our tinnitus patients compared to matched controls. It is possible that the absence of any hearing loss in our tinnitus population may be one reason for the different results. In addition, the MR technique and analytical method may be less sensitive for detecting subtle structural changes.

In this study, main effects of chronic tinnitus were primarily in auditory cortex and prefrontal cortex. Our tinnitus patient group exhibited decreased rCBF primarily in the right STG, which was associated with THQ scores. Dysfunction of the temporal gyrus is associated with affective disturbance, which has been proved to be linked with migraine [24-26]. The tinnitus comorbid with migraine may lead to more complex dysfunction in the cortico-limbic network. Moreover, prior fMRI researches have revealed the associations between aberrant neuronal activity and functional connectivity of the STG and tinnitus distress[27, 28]. However, Emmert et al. found no significant rCBF alterations within the auditory cortex in tinnitus patients using ASL [14]. Moreover, previous studies using SPECT or PET did not detect reduced cerebral perfusion in primary auditory cortex [11, 10, 9], which was different from our current results. We infer that different sample sizes and imaging modalities may contribute to the discrepancy. Furthermore, the prefrontal cortex, including the MFG and SFG, exhibited decreased $\mathrm{rCBF}$ in tinnitus patients compared with nontinnitus controls. The prefrontal cortex plays a critical role in emotional processing and executive function [29]. Disrupted brain activity was observed within the executive control of attention network, including the MFG and SFG[8]. Previous resting-state fMRI studies have also pointed out the abnormalities of the prefrontal cortex could act as a direct mechanism of tinnitus chronification [30, 31]. Therefore, these findings suggested that the $\mathrm{rCBF}$ alterations in the prefrontal cortex may be one important brain characteristic of chronic tinnitus. Nevertheless, the specific clinical implication for reduced $\mathrm{rCBF}$ in the MFG and SFG is unclear and requires to be further investigated.

Previous studies showed that patients with migraine exhibited reduced perfusion in temporal and prefrontal cortex $[15,16]$. Our data support and extend these findings by identifying a significant effect of migraine on tinnitus for $\mathrm{rCBF}$ in the STG and MFG, indicating that the existence of migraine accelerates the decrease in $\mathrm{rCBF}$ in the setting of tinnitus, resulting in impaired attention and executive function. In addition, the severity of migraine is negatively associated with decreased rCBF in tinnitus patients, which was consistent with some researches about migraine without aura attacks [15-17]. We speculate that increased severity of migraine will aggravate the tinnitus distress and severity, reflecting the
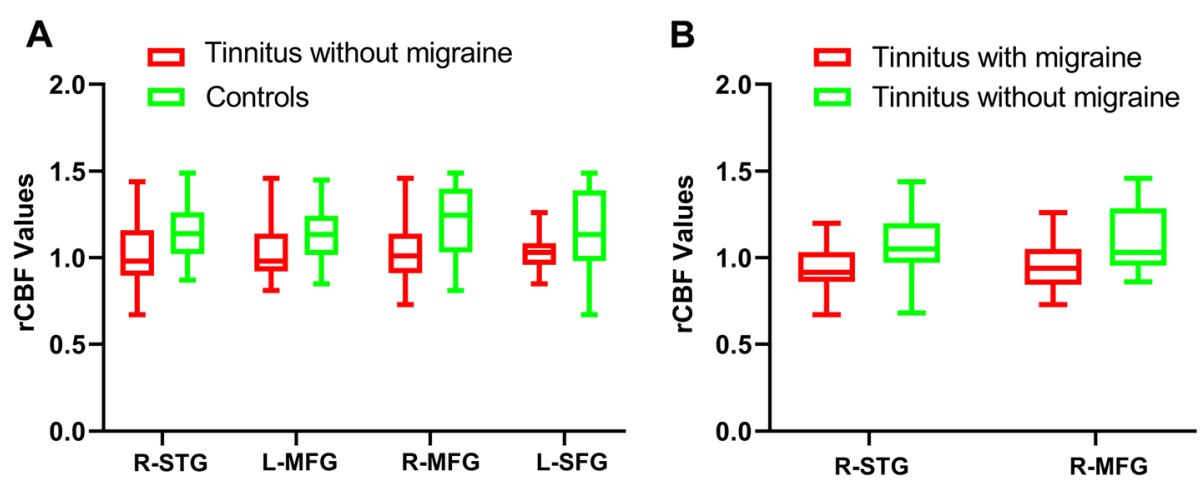

Fig. 3 The rCBF values for different groups. (A) The rCBF values of the chronic tinnitus patients without migraine and non-tinnitus controls in the right STG, left MFG, right MFG, and left SFG $(P<0.01)$. (B) The interaction effect of migraine on tinnitus in the right STG and right MFG $(P<0.01)$ 

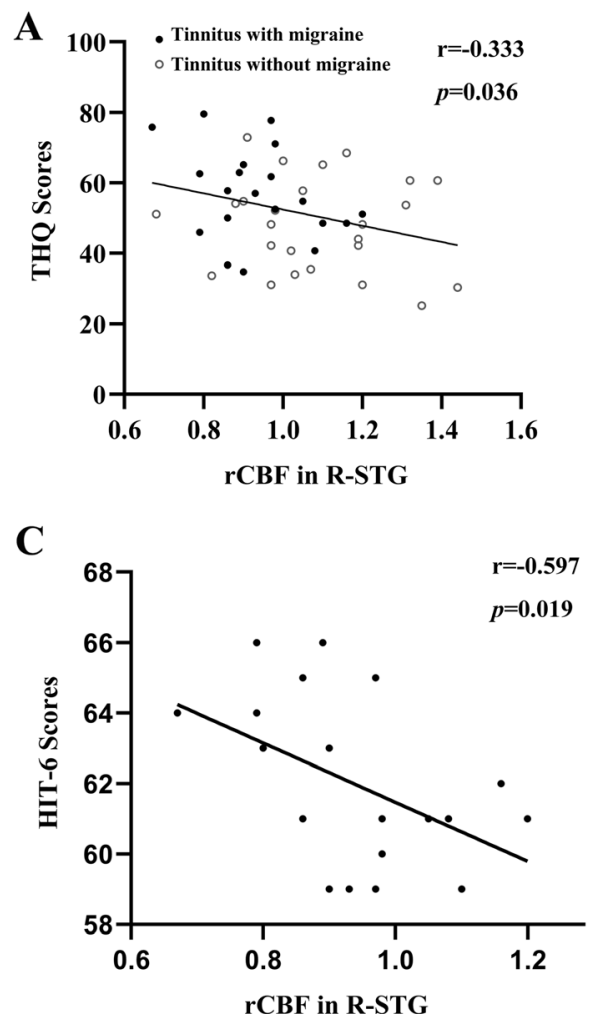
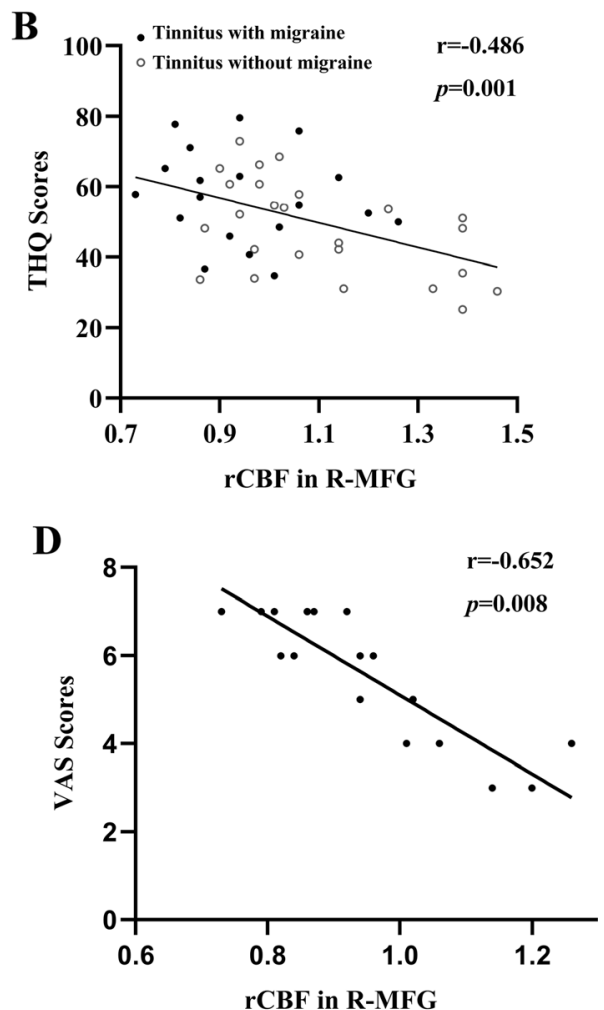

Fig. 4 The significant correlations between the rCBF changes and the clinical data. (A) rCBF in the right STG was negatively associated with THQ scores $(r=-0.333, p=0.036)$ in tinnitus patients with and without migraine; (B) rCBF in the right MFG was negatively associated with THQ scores $(r=-0.486, p=0.001)$ in tinnitus patients with and without migraine; (C) rCBF in the right STG was inversely correlated with the HIT-6 scores ( $r=-$ $0.597, p=0.019)$ in tinnitus patients with migraine; (D) rCBF in the right MFG was inversely correlated with the VAS scores $(r=-0.652, p=0.008)$ in tinnitus patients with migraine

aberrant perfusion in specific brain regions. Nevertheless, the association of the migraine with the chronic tinnitus has not been substantially elucidated and also needs to be corroborated in future studies.

Several limitations should be acknowledged in the current study. Firstly, due to the strict inclusion and exclusion criteria, our sample size was not large enough that may affect the statistical reliability of our results. We will further expand the sample size and obtain more reliable results in future studies. Secondly, the characteristics of migraine were just assessed using HIT-6 and VAS scores in this study. More neuropsychological tests are needed to assess the symptom of the migraine, such as the Migraine Disability Assessment Questionnaire (MIDAS) [32] and Visual Light Sensitivity Questionnaire-8 (VLSQ-8) [33]. Furthermore, although we try to diminish the MR scanner noise using earplugs, the tinnitus patients cannot be completely prevented from scanner noise that probably affects the rCBF perfusion to varying degree. This confounding factor should be taken into account for all perfusion fMRI studies related to the auditory system. Finally, we only measured the $\mathrm{rCBF}$ changes within each brain regions but did not prove the possibility of rCBF functional connectivity among different brain areas. A data- driven approach to whole-brain $\mathrm{rCBF}$ connectivity analysis will be considered in our future study.

In conclusion, this study provided evidence that chronic tinnitus patients exhibited reduced $\mathrm{rCBF}$ in auditory and prefrontal cortex, and that decreased $\mathrm{rCBF}$ in tinnitus patients was associated with tinnitus severity. Migraine may exacerbate $\mathrm{rCBF}$ reduction in tinnitus patients, and the severity of migraine was negatively associated with $\mathrm{rCBF}$ levels. These findings suggest that abnormalities in $\mathrm{rCBF}$ perfusion may contribute to the pathophysiological mechanisms of chronic tinnitus with migraine condition. The co-occurrence of tinnitus and migraine is not purely coincidental but both disorders may be linked by shared neuropathological mechanisms.

\section{Abbreviations}

fMRI: functional magnetic resonance imaging; SPECT: single-photon emission computed tomography; PET: positron emission tomography; CBF: cerebral blood flow; ASL: arterial spin labeling; GM: gray matter; WM: white matter; THQ: Tinnitus Handicap Questionnaires; PTA: pure tone audiometry; SDS: Self-Rating Depression Scale; SAS: Self-Rating Anxiety Scale; VAS: visual analogue scale; HIT-6: Headache Impact Test-6; FDR: false discovery rate; FWE: family-wise error; STG: superior temporal gyrus; MFG: middle frontal gyrus; SFG: superior frontal gyrus 


\section{Acknowledgements}

We thank all the patients and controls for their participation in the study.

\section{Authors' contributions}

$Z-G X$ and $J-J X$ designed the study, performed the experiments, and wrote the manuscript. Y- $\mathrm{C} \mathrm{C}$ and $\mathrm{JH}$ performed the experiments and analyzed the data. $Y W$ and $Y X$ revised the manuscript. All authors read and approved the final manuscript.

\section{Funding}

This work was supported by the 14th "Six Talent Peaks" Project of Jiangsu Province (No. YY-079) and Medical Science and Technology Development Foundation of Nanjing Department of Health (No. ZKX20037).

\section{Availability of data and materials}

Clinical, neuroimaging and statistical data will be available upon request from any qualified investigator.

\section{Declarations}

\section{Ethics approval and consent to participate}

Study protocol was approved by the Research Ethics Committee of the Jiangsu Province Hospital prior to study initiation. All the subjects provided written informed consent before any study procedures.

\section{Consent for publication}

Not applicable.

\section{Competing interest}

The authors declare that they have no competing interests.

\section{Author details}

'Department of Otolaryngology, Nanjing Pukou Central Hospital, Pukou Branch Hospital of Jiangsu Province Hospital, No.166, Shanghe Road, 211899 Nanjing, China. ${ }^{2}$ Department of Otolaryngology, Nanjing First Hospital, Nanjing Medical University, No.68, Changle Road, 210006 Nanjing, China. ${ }^{3}$ Department of Radiology, Nanjing First Hospital, Nanjing Medical University, 210006 Nanjing, China.

\section{Received: 27 April 2021 Accepted: 2 June 2021}

\section{Published online: 29 June 2021}

\section{References}

1. Bauer CA. Tinnitus (2018) N Engl J Med 378(13):1224-1231. doi:https://doi. org/10.1056/NEJMcp1506631

2. Langguth B, Kreuzer PM, Kleinjung T, De Ridder D (2013) Tinnitus: causes and clinical management. Lancet Neurol 12(9):920-930. doi:https://doi.org/1 0.1016/S1474-4422(13)70160-1

3. Bhatt JM, Bhattacharyya N, Lin HW (2017) Relationships between tinnitus and the prevalence of anxiety and depression. Laryngoscope 127(2):466469

4. Kreuzer PM, Landgrebe M, Vielsmeier V, Kleinjung T, De Ridder D, Langguth B (2014) Trauma-associated tinnitus. J Head Trauma Rehabil 29(5):432-442. doi:https://doi.org/10.1097/HTR.0b013e31829d3129

5. Langguth $B$, Hund V, Busch V, Jurgens TP, Lainez JM, Landgrebe M et a (2015) Tinnitus Headache BioMed research international 2015:797416. doi: https://doi.org/10.1155/2015/797416

6. de Tommaso M, Ambrosini A, Brighina F, Coppola G, Perrotta A, Pierelli F et al (2014) Altered processing of sensory stimuli in patients with migraine. Nature reviews Neurology 10(3):144-155. doi:https://doi.org/10.1038/ nrneurol.2014.14

7. De Ridder D, Vanneste S, Langguth B, Llinas R (2015) Thalamocortical Dysrhythmia: A Theoretical Update in Tinnitus. Front Neurol 6:124. doi: https://doi.org/10.3389/fneur.2015.00124

8. Vanneste S, De Ridder D (2012) The auditory and non-auditory brain areas involved in tinnitus. An emergent property of multiple parallel overlapping subnetworks. Front Syst Neurosci 6:31. doi:https://doi.org/10.3389/fnsys.2 012.00031

9. Laureano MR, Onishi ET, Bressan RA, Castiglioni MLV, Batista IR, Reis MA et al. Memory networks in tinnitus: a functional brain image study. PLoS One. 2014;9(2):e87839. https://doi.org/10.1371/journal.pone.0087839.
10. Farhadi M, Mahmoudian S, Saddadi F, Karimian AR, Mirzaee M, Ahmadizadeh M et al (2010) Functional brain abnormalities localized in 55 chronic tinnitus patients: fusion of SPECT coincidence imaging and MRI. Journal of Cerebral Blood Flow Metabolism 30(4):864-870

11. Geven L, De Kleine E, Willemsen A, Van Dijk P (2014) Asymmetry in primary auditory cortex activity in tinnitus patients and controls. Neuroscience 256: $117-125$

12. Detre JA, Leigh JS, Williams DS, Koretsky AP (1992) Perfusion imaging. Magnetic resonance in medicine 23(1):37-45

13. Ho ML (2018) Arterial spin labeling: Clinical applications. Journal of neuroradiology = Journal de neuroradiologie 45(5):276-289. doi:https://doi. org/10.1016/.jneurad.2018.06.003

14. Emmert K, Kopel R, Koush Y, Maire R, Senn P, Van De Ville D et al (2017) Continuous vs. intermittent neurofeedback to regulate auditory cortex activity of tinnitus patients using real-time fMRI - A pilot study. Neurolmage Clinical 14:97-104. doi:https://doi.org/10.1016/j.nicl.2016.12.023

15. Chen Z, Chen X, Liu M, Ma L, Yu S (2018) Evaluation of gray matter perfusion in episodic migraine using voxel-wise comparison of $3 \mathrm{D}$ pseudocontinuous arterial spin labeling. J Headache Pain 19(1):36. doi:https://doi. org/10.1186/s10194-018-0866-y

16. Hu B, Yu Y, Dai YJ, Feng JH, Yan LF, Sun Q et al (2019) Multi-modal MRI Reveals the Neurovascular Coupling Dysfunction in Chronic Migraine. Neuroscience 419:72-82. doi:https://doi.org/10.1016/.neuroscience.2019.09.022

17. Gil-Gouveia R, Pinto J, Figueiredo P, Vilela PF, Martins IP (2017) An Arterial Spin Labeling MRI Perfusion Study of Migraine without Aura Attacks. Front Neurol 8:280. doi:https://doi.org/10.3389/fneur.2017.00280

18. The International Classification of Headache Disorders, 3rd edition (beta version). Cephalalgia: an international journal of headache. 2013;33(9):629_ 808. doi:https://doi.org/10.1177/0333102413485658

19. Kuk FK, Tyler RS, Russell D, Jordan H (1990) The psychometric properties of a tinnitus handicap questionnaire. Ear hearing 11(6):434-445

20. Ashburner J, Friston KJ (2005) Unified segmentation. Neurolmage 26(3):839-851

21. Wang Z (2012) Improving cerebral blood flow quantification for arterial spin labeled perfusion MRI by removing residual motion artifacts and global signal fluctuations. Magn Reson Imaging 30(10):1409-1415

22. Aslan S, Lu H (2010) On the sensitivity of ASL MRI in detecting regional differences in cerebral blood flow. Magn Reson Imaging 28(7):928-935

23. Adjamian P. Hall DA, Palmer AR, Allan TW, Langers DR (2014) Neuroanatomical abnormalities in chronic tinnitus in the human brain. Neurosci Biobehav Rev 45:119-133. doi:https://doi.org/10.1016/j.neubiorev.2 014.05 .013

24. Dumkrieger G, Chong CD, Ross K, Berisha V, Schwedt TJ (2019) Static and dynamic functional connectivity differences between migraine and persistent post-traumatic headache: A resting-state magnetic resonance imaging study. Cephalalgia: an international journal of headache 39(11): 1366-1381. doi:https://doi.org/10.1177/0333102419847728

25. Chen C, Yan M, Yu Y, Ke J, Xu C, Guo X et al (2019) Alterations in Regional Homogeneity Assessed by fMRI in Patients with Migraine Without Aura. Journal of medical systems 43(9):298. doi:https://doi.org/10.1007/s10916-01 $9-1425-z$

26. Chen Z, Chen X, Liu M, Liu S, Ma L, Yu S (2017) Disrupted functional connectivity of periaqueductal gray subregions in episodic migraine. J Headache Pain 18(1):36. doi:https://doi.org/10.1186/s10194-017-0747-9

27. Cheng S, Xu G, Zhou J, Qu Y, Li Z, He Z et al (2020) A Multimodal MetaAnalysis of Structural and Functional Changes in the Brain of Tinnitus. Front Hum Neurosci 14:28. doi:https://doi.org/10.3389/fnhum.2020.00028

28. Minami SB, Oishi N, Watabe T, Uno K, Ogawa K (2018) Auditory Related Resting State fMRI Functional Connectivity in Tinnitus Patients: Tinnitus Diagnosis Performance. Otology \& neurotology: official publication of the American Otological Society. American Neurotology Society [and] European Academy of Otology Neurotology 39(1):1-5. doi:https://doi.org/10.1097/ma 0.0000000000001626

29. Etkin A, Egner T, Kalisch R (2011) Emotional processing in anterior cingulate and medial prefrontal cortex. Trends Cogn Sci 15(2):85-93

30. Schmidt SA, Akrofi K, Carpenter-Thompson JR, Husain FT (2013) Default mode, dorsal attention and auditory resting state networks exhibit differential functional connectivity in tinnitus and hearing loss. PloS one 8(10):e76488. doi:https://doi.org/10.1371/journal.pone.0076488

31. Ueyama T, Donishi T, Ukai S, Ikeda Y, Hotomi M, Yamanaka N et al (2013) Brain regions responsible for tinnitus distress and loudness: a resting-state fMRI study. PloS one 8(6):e67778 
32. Stewart WF, Lipton RB, Whyte J, Dowson A, Kolodner K, Liberman JN et al (1999) An international study to assess reliability of the Migraine Disability Assessment (MIDAS) score. Neurology 53(5):988-994. doi:https://doi.org/1 $0.1212 / \mathrm{wnl} .53 .5 .988$

33. Verriotto JD, Gonzalez A, Aguilar MC, Parel J-MA, Feuer WJ, Smith AR et al (2017) New methods for quantification of visual photosensitivity threshold and symptoms. Translational vision science technology 6(4):18-

\section{Publisher's Note}

Springer Nature remains neutral with regard to jurisdictional claims in published maps and institutional affiliations.

Ready to submit your research? Choose BMC and benefit from:

- fast, convenient online submission

- thorough peer review by experienced researchers in your field

- rapid publication on acceptance

- support for research data, including large and complex data types

- gold Open Access which fosters wider collaboration and increased citations

- maximum visibility for your research: over $100 \mathrm{M}$ website views per year

At $\mathrm{BMC}$, research is always in progress.

Learn more biomedcentral.com/submissions 